\title{
The paradigm of tumor shrinkage and rapid liver remnant hypertrophy for conversion of initially unresectable colorectal liver metastasis: a case report and literature review
}

\author{
Nan Xiao ${ }^{1 \dagger}$, Kailin $\mathrm{Yu}^{1+}$, Shaojun $\mathrm{Yu}^{1}$, Jianjun $\mathrm{Wu}^{2}$, Jian Wang ${ }^{1}$, Siyang Shan ${ }^{1}$, Shuchun Zheng ${ }^{1}$, Liuhong Wang ${ }^{2}$, \\ Jianwei Wang ${ }^{1 *}$ and Shuyou Peng ${ }^{3}$
}

\begin{abstract}
Background: For colorectal liver metastasis (CRLM) patients, hepatic resection is currently the sole cure offering the chance of long-term survival. Tumor shrinkage and planned liver remnant hypertrophy are the two key strategies for conversion of initially unresectable CRLM. First conducted in 2012, associated liver partition and portal vein ligation for staged hepatectomy (ALPPS) allows rapid liver growth. As a means to induce hypertrophy, portal vein embolization (PVE) has been widely applied before extending hepatectomy. Recently, Peng et al. present a new approach of terminal branches portal vein embolization (TBPVE), offering an efficient way to amplify FLR and making chances for surgery in 2 weeks.
\end{abstract}

Case presentation: We reported a 61-year-old woman with synchronous hepatic metastasized carcinoma of the colon sigmoideum underwent TBPVE after 6 cycles of neoadjuvant therapy in order to perform a planned right trisectionectomy. Rapid liver remnant hypertrophy and remarkable tumor shrinkage were achieved, and laparoscopic sigmoidectomy and right trisectionectomy were successfully performed. The postsurgical course was uneventful and 7 months of recurrence-free survival have been witnessed.

Conclusions: The dual tactics of tumor shrinkage and planned rapid liver remnant hypertrophy will make concerted efforts to further increase the clinical candidacy for curative resection, which are valuable for further investigation.

Keywords: Tumor shrinkage, Liver remnant hypertrophy, Unresectable colorectal liver metastasis, Preoperative chemotherapy

\section{Background}

Colorectal cancer is the third leading cause of malignancy-related death worldwide. The liver is the most common metastatic site of colorectal cancer and liver metastasis occurs in $20-40 \%$ of patients at the time of primary diagnosis [1]. Liver resection is the sole curative approach to colorectal liver metastasis (CRLM).

\footnotetext{
* Correspondence: sypzju@zju.edu.cn

${ }^{\dagger}$ Equal contributors

${ }^{1}$ Department of Surgical Oncology, Second Affiliated Hospital of Zhejiang

University School of Medicine, Hangzhou 310009, China

Full list of author information is available at the end of the article
}

Nevertheless, only less than $25 \%$ of patients with CRLM are eligible for curative resection, of which insufficient future liver remnant (FLR) volume is the main cause [2]. Currently, systemic therapy is the only established treatment for patients with unresectable CRLM. Tumor shrinkage and liver remnant hypertrophy are the two key strategies for conversion of initially unresectable CRLM.

First conducted in 2012, associated liver partition and portal vein ligation for staged hepatectomy (ALPPS) allows rapid liver growth in a short time and has a strong impact on surgery for liver cancer. However, high morbidity and 
mortality after the surgery cannot be ignored. Classic portal vein embolization (PVE) has been widely applied before extending hepatectomy, but the slow growth of FLR renders a longish waiting time of 4-6 weeks between PVE and liver resection, which embraces the risk of tumor progression. Recently, Peng et al. [3] presented a new approach of terminal branches portal vein embolization liver partition for planned hepatectomy (TELPP), offering an efficient way to amplify the FLR and making chances for surgery in 2 weeks.

\section{Case presentation}

A 61-year-old woman was referred to our department because of synchronous hepatic metastasized carcinoma of the colon sigmoideum. Physical examination revealed no significant abnormalities. Blood investigations were within normal range except for mild anemia and serum carcinoembryonic antigen level of $10 \mathrm{ng} / \mathrm{ml}$. Fecal occult blood test was strongly positive.

Colonoscopy found a sigmoid lesion $20 \mathrm{~cm}$ from the anus, and pathological result of the biopsy specimen demonstrated a moderately differentiated adenocarcinoma. The gene detection found no mutation in KRAS, NRAS, and BRAF. Magnetic resonance imaging of the liver revealed multiple metastases and the largest measured $15 \mathrm{~cm} \times 11 \mathrm{~cm} \times 13 \mathrm{~cm}$ (staging according the seventh edition of the UICC: cT3, cN1, cM1a) (Fig. 1).

The multi-disciplinary team (MDT) suggested a conversion strategy consisting of preoperative FOLFIRI (irinotecan, fluoruracil) regimen with cetuximab for the initially unresectable colorectal liver metastasis. If the conversion of liver metastasis was achieved, a right trisectionectomy would be performed.

After 6 cycles, the liver metastasis significantly shrank (staging according to RECIST version 1.1: PR), but the largest metastasis was adjacent to the left branch of portal vein (Fig. 2). The CT volumetry revealed that total liver volume (TLV) was $1495 \mathrm{ml}$, and the volume of the future liver remnant (FLR) was $415 \mathrm{ml}$, which was below the volume cutoff value for safe resection (more than $40 \%$ of TLV) [4]. So, according to MDT, terminal branches portal vein embolization (TBPVE) was performed to block the anterior and posterior branch of the right portal vein as previously reported [3] for sufficient hypertrophy of the left lateral lobe (Fig. 3).

Five days after the TBPVE, the FLR was $513 \mathrm{ml}(47.5 \%$ of TLV). On the 11th day, the FLR was $550 \mathrm{ml}(50.9 \%$ of TLV) (Fig. 4) and laparoscopic sigmoidectomy and right trisectionectomy were performed (Fig. 5). There were no intraoperative complications, and the histology of the FLR showed a low-grade steatohepatitis after chemotherapy. The postoperative histology revealed $\mathrm{pT} 3, \mathrm{pN} 2 \mathrm{~b}$ (11/17), pM1, L0, V1, G2, and R0 resection margin. The postsurgical course was uneventful and the patient continued to undertake 6 cycles of systemic therapy of FOLFIRI with cetuximab. Heretofore, 7 months of recurrence-free survival have been witnessed.

\section{Discussion}

For CRLM patients, hepatic resection is the only treatment with a curative intent offering the chance of long-term survival at present. Nevertheless, at the time of diagnosis, only fewer than $25 \%$ of CRLM patients are eligible for liver resection. The main limitations to the resection are inadequate future liver remnant (FLR) volume and poor oncological prognosis of advanced diseases. The former increases the risk of postoperative liver failure, while the latter is associated with early recurrence.

The past few decades have seen surgeons struggling to achieve rapid liver remnant hypertrophy. As a classic way to induce hypertrophy of the FLR, PVE is now routinely applied to improve the rate of R0 resection. But it takes 3-8 weeks to expand the volume of FLR by up to $40 \%$ and the second stage of the surgery is not always accomplished [5]. First performed by Schnitzbauer in 2012, ALPPS led to $74 \%$ increase of the FLR in a mean time of 9 days, making initially unresectable hepatic lesions resectable. Despite its advantages, ALPPS caused high morbidity (64\%) and liver-related mortality (12\%), which has been evoking violent controversy [6].

To overcome the aforementioned disadvantages, Peng et al. [3] presented a new approach of TBPVE, which deflowered the essence of PVE and ALPPS. It is reported that this new technique could induce rapid liver remnant

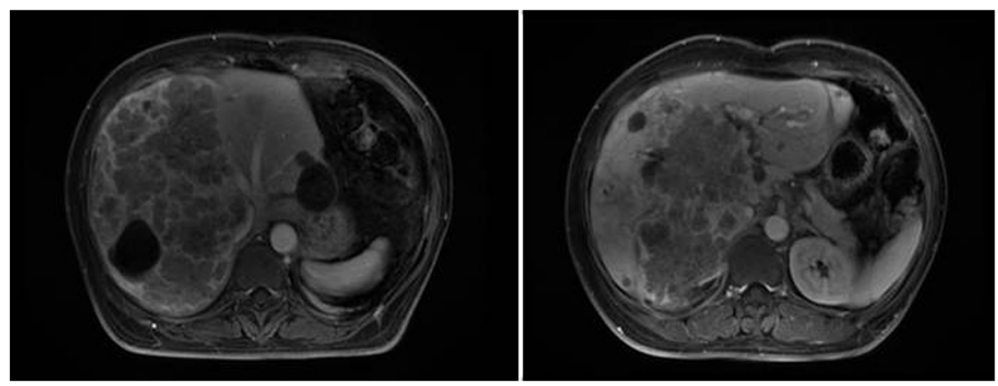

Fig. $1 \mathrm{MRl}$ imaging of the mass revealed tumor distributed at the right hepatic trisection, adjacent to the left branch of portal vein at primary diagnosis 


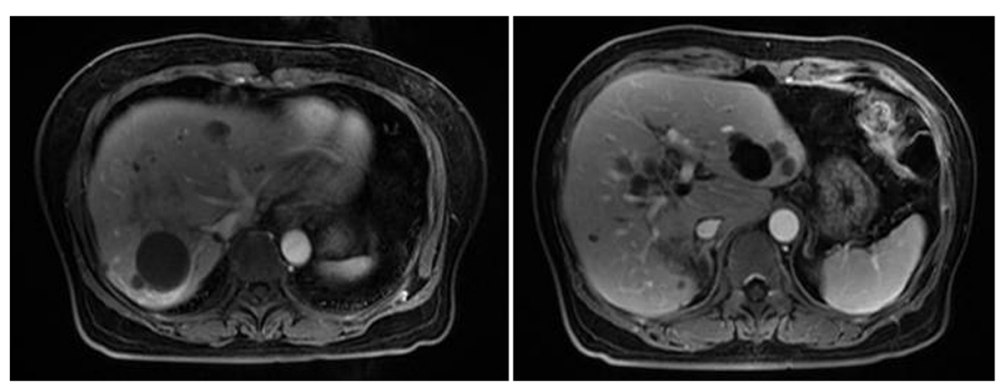

Fig. 2 Tumor shrunk significantly after 6 cycles of FOLFIRI with cetuximab

hypertrophy and make chances for surgery in 14 days. Increasing FLR by $33 \%$ to $68 \%$, four cases of huge hepatocellular carcinoma with liver cirrhosis acquired chances of right hepatectomy 2 weeks after the TBPVE. His group recently reported the safety and efficacy data of tentative application of TBPVE liver partition for planned hepatectomy (TELPP). Similar to that of ALPPS, the philosophy behind TBPVE is the thorough separation of the left and right hemi-liver (ramus communicans of the right and left portal vein). Moreover, complete and enduring embolism of the terminal branches to segment $\mathrm{V}$, VIII or IV is indispensible in order to rapidly promote the regeneration of remnant liver parenchyma. Technically, to achieve this goal, they first embolized the terminal branches with lipiodol and cyanoacrylate, then blocked the main branch of portal vein with coils. Compared with classic PVE, the new technique could increase the FLR more significantly in a short period of time. The specific physiopathological mechanisms underlying this enhanced liver remnant hypertrophy are still unconfirmed. The explanation for this phenomenon might be that, in comparison to PVE, TBPVE attains more thorough partitions of communicating branches between the planned resected segments and FLR, which lead to increasing portal flow and hepatotropic factors to the FLR [7].

The use of first-line chemotherapy to shrink hepatic metastases is an ideal strategy for patients with initially unresectable CLRM, but only about $20 \%$ of the patients with initially unresectable liver-limited metastases become resectable after chemotherapy [8]. Considering the low tumor response rate yielded by second-line chemotherapy after the failure of first-line regimen [9], we believe TBPVE could be a better choice for these patients as a fast and efficient way to amplify FLR and create chances for surgery in a short time.

There is growing evidence that PVE not only does stimulate the growth of FLR but also induces significant tumor growth in patients with CRLM $[10,11]$. The longish time between PVE and liver resection required to achieve adequate FLR volume embraces the risk of progressive disease [12]. Moreover, potential promotion of tumor growth after PVE and consequent acceleration of tumor progression in the waiting time are primary concerns possibly confining the use of PVE in patients with multifocal tumors [13]. A recent meta-analysis [5] reviewing 44 publications and including 1791 patients undergoing PVE demonstrated that the hypertrophy response was insufficient in 51 patients (2.8\%) to perform liver resection and $6.1 \%$ of those patients treated ultimately were not able to undergo resection because of local tumor progression after PVE. Another study by Fischer et al. [14] based on 208 tumors measured in 64 patients found that, without post-PVE chemotherapy, $34.2 \%$ of the liver lesions progressed. However, there was a remarkable lower risk of tumor progression $(18.9 \%, p=.03)$ when chemotherapy was applied.

Consequently, it has sparked controversy over whether preoperative chemotherapy could cooperate with PVE.

Surely, there are sufficient practical and academic justifications for preoperative chemotherapy. Approximately
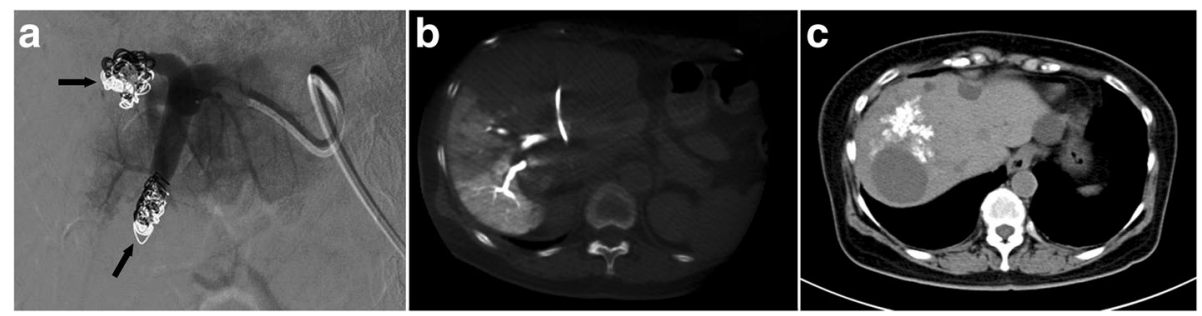

Fig. 3 Effect of terminal branch portal vein embolization. a Post-embolization venogram with coils visible in the anterior and posterior right portal vein (black arrows). b CT scan showing embolized terminal branches of the right portal vein. c CT scan showing lipiodol deposition 


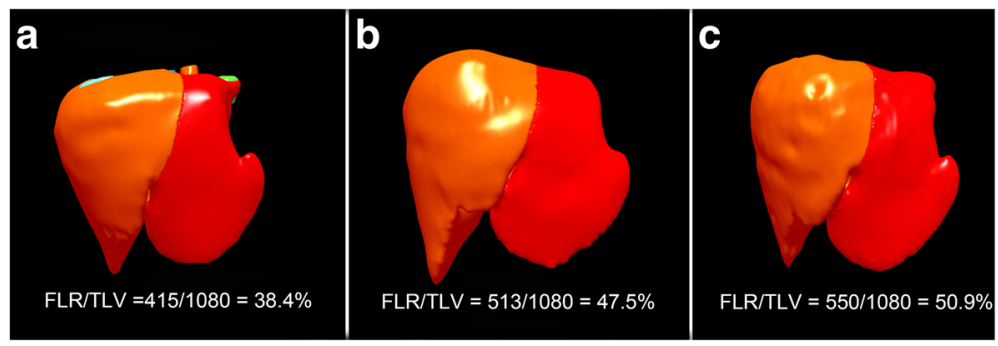

Fig. 4 Volume of the left lateral external lobe of the liver. a Before the TBPVE. $\mathbf{b}$ Hypertrophy on the 5th and $\mathbf{c} 11$ th day after the TBPVE

$15 \%$ of patients with initially unresectable hepatic colorectal metastases are now conventionally converted to resectable cases by chemotherapy. The chemotherapy and biologic treatments given while the tumor is in situ can not only help to decide the appropriate therapies after surgery but also eliminate microscopic tumor cells.

Nevertheless, it was suggested that preoperative chemotherapy would hinder liver regeneration [15] and increase postoperative complications [16]. Therefore, chemotherapy was interrupted several weeks before embolization, leaving the potential for tumor progression at liberty.

Prolonged peri-procedure chemotherapy has been associated with reduced hypertrophy $[17,18]$. Yet, some studies [19-21] were unable to show any influence of chemotherapy on liver regeneration after PVE. On the other hand, Covey et al. [22] in a study with a series of 100 patients concluded that liver could still hypertrophy in a toxic environment and preoperative chemotherapy during PVE had no negative effects on liver regeneration. Most recently, Fischer et al. [14] reported the combination of PVE and chemotherapy was not only effective in terms of liver hypertrophy but also related to retarded tumor growth and improved long-term survival. Additionally, it is indicated that short chemical-free intervals (CFI) improved outcomes, whereas long CFI resulted in poor oncological endings. Kambakamba et al. [23] found that short CFI is closely associated with significantly better prognosis in terms of overall survival and disease-free survival based on 74 patients suffering from CRLM who received operations. Spelt and his colleagues [24] also came to the conclusion that long intervals between the end of chemotherapy and PVE could enhance tumor progression in CRLM patients.

\section{Conclusion}

In conclusion, TBPVE was a promising approach to shorten the waiting time between embolization and surgery. Preliminary study indicated that TBPVE could rival ALPPS in respect of inducing rapid liver remnant hypertrophy and, meanwhile, be on a par with PVE in terms of postoperation complications. To confirm its safety and efficacy, further large-scale and multi-centered studies are needed.

The threat of progressive disease after PVE highlights the value of minimizing the waiting time between PVE and resection and of devising therapeutic strategies using preoperative chemotherapy to control tumor growth after PVE. Besides, given that the liver can still regenerate when cytotoxic chemotherapy is administered, the modality combining preoperative chemotherapy with TBPVE would attain lesion shrinkage while also achieving rapid liver remnant expansion. The dual tactics of tumor shrinkage and planned rapid liver remnant hypertrophy will make concerted efforts to further increase the clinical candidacy for curative resection, which are valuable for further investigation.

\section{Abbreviations}

ALPPS: Associated liver partition and portal vein ligation for staged hepatectomy; CFI: Chemical-free intervals; CRLM: Colorectal liver metastasis; FLR: Future liver
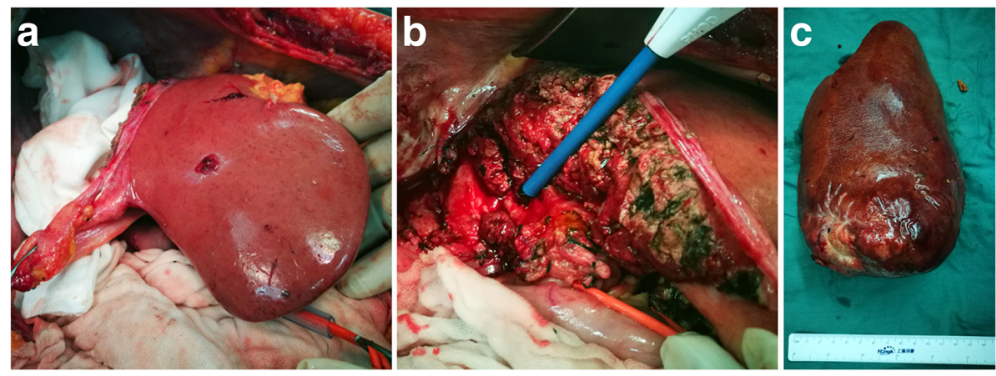

Fig. 5 Right trisectionectomy a hypertrophy of the left lateral external lobe of the liver $\mathbf{b}$ pars sagitalis of the left branch of portal vein $\mathbf{c}$ right trisectionectomy specimen 
remnant; MDT: Multi-disciplinary team; PVE: Portal vein embolization; TBPVE: Terminal branches portal vein embolization; TELPP: Terminal branches portal vein embolization liver partition for planned hepatectomy; TLV: Total liver volume

\section{Acknowledgements}

Not applicable.

\section{Funding}

This study was supported by the Natural Science Fund of China under Grant No. 81672364 and the Zhejiang Province Natural Science Foundation of China under Grant No. LY15H160023.

\section{Availability of data and materials}

Data sharing is not applicable to this article as no datasets were generated or analyzed during the current study.

\section{Authors' contributions}

NX and KLY are the co-first author. JWW is the corresponding author of the manuscript. SJY, JJW, JW and LHW collected the patient's data and provided the figures. SYS, SCZ, and SYP were involved in drafting and revising the manuscript. All authors read and approved the final manuscript.

\section{Ethics approval and consent to participate}

Ethical approval was given by the medical ethics committee of Second Affiliated Hospital School of Medicine, Zhejiang University.

\section{Consent for publication}

Written informed consent for publication of this case report and the associated images were obtained from the patient.

\section{Competing interests}

The authors declare that they have no competing interests.

\section{Publisher's Note}

Springer Nature remains neutral with regard to jurisdictional claims in published maps and institutional affiliations.

\section{Author details}

'Department of Surgical Oncology, Second Affiliated Hospital of Zhejiang University School of Medicine, Hangzhou 310009, China. ${ }^{2}$ Department of Radiology and Intervention, Second Affiliated Hospital of Zhejiang University School of Medicine, Hangzhou, China. ${ }^{3}$ Department of General Surgery, Second Affiliated Hospital of Zhejiang University School of Medicine, Hangzhou, China.

Received: 18 April 2017 Accepted: 22 July 2017

Published online: 03 August 2017

\section{References}

1. Siegel RL, Miller KD, Jemal A. Cancer statistics, 2016. CA Cancer J Clin. 2016;66:7-30.

2. Lam W, Laurence JM, Johnston E, Hollands MJ, Pleass HC, Richardson AJ. A systematic review of two-stage hepatectomy in patients with initially unresectable colorectal liver metastases. HPB (Oxford). 2013;15:483-91.

3. Peng SY, Huang CY, Li JT, Zhang YY, He XW, Wang YF, Hong DF, Cai XJ. Terminal branches portal vein embolization for planed hepatectomy. Zhonghua Wai Ke Za Zhi. 2016;54(9):664-8.

4. Abdalla EK, Adam R, Bilchik AJ, Jaeck D, Vauthey JN, Mahvi D. Improving resectability of hepatic colorectal metastases: expert consensus statement. Ann Surg Oncol. 2006;13:1271-80.

5. van Lienden KP, van den Esschert JW, de Graaf W, Bipat S, Lameris JS, van Gulik TM, van Delden OM. Portal vein embolization before liver resection: a systematic review. Cardiovasc Intervent Radiol. 2013;36:25-34.

6. Schnitzbauer AA, Lang SA, Goessmann H, Nadalin S, Baumgart J, Farkas SA, Fichtner-Feigl S, Lorf T, Goralcyk A, Horbelt R, et al. Right portal vein ligation combined with in situ splitting induces rapid left lateral liver lobe hypertrophy enabling 2-staged extended right hepatic resection in small-for-size settings. Ann Surg. 2012;255:405-14.
7. Yokoyama Y, Nagino M, Nimura Y. Mechanisms of hepatic regeneration following portal vein embolization and partial hepatectomy: a review. World J Surg. 2007;31:367-74.

8. Maeda Y, Shinohara T, Nagatsu A, Futakawa N, Hamada T. Long-term outcomes of conversion hepatectomy for initially unresectable colorectal liver metastases. Ann Surg Oncol. 2016;23(Suppl 2):S242-8.

9. Mocellin S, Baretta Z, Roque IFM, Sola I, Martin-Richard M, Hallum S, Bonfill Cosp X. Second-line systemic therapy for metastatic colorectal cancer. Cochrane Database Syst Rev. 2017;1:Cd006875.

10. Hoekstra LT, van Lienden KP, Doets A, Busch OR, Gouma DJ, van Gulik TM. Tumor progression after preoperative portal vein embolization. Ann Surg. 2012;256:812-7. discussion 817-818

11. Simoneau E, Aljiffry M, Salman A, Abualhassan N, Cabrera T, Valenti D, E Baage A, Jamal M, Kavan P, Al-Abbad S, et al. Portal vein embolization stimulates tumour growth in patients with colorectal cancer liver metastases. HPB (Oxford). 2012;14:461-8.

12. de Graaf W, van den Esschert JW, van Lienden KP, van Gulik TM. Induction of tumor growth after preoperative portal vein embolization: is it a real problem? Ann Surg Oncol. 2009;16:423-30.

13. van Gulik TM, van den Esschert JW, de Graaf W, van Lienden KP, Busch OR, Heger M, van Delden OM, Lameris JS, Gouma DJ. Controversies in the use of portal vein embolization. Dig Surg. 2008:25:436-44.

14. Fischer C, Melstrom LG, Arnaoutakis D, Jarnagin W, Brown K, D'Angelica M, Covey A, DeMatteo R, Allen P, Kingham TP, et al. Chemotherapy after portal vein embolization to protect against tumor growth during liver hypertrophy before hepatectomy. JAMA Surg. 2013:148:1103-8.

15. Kremer M, Manzini G, Hristov B, Polychronidis G, Mokry T, Sommer CM, Mehrabi A, Weitz J, Buchler MW, Schemmer P. Impact of neoadjuvant chemotherapy on hypertrophy of the future liver remnant after associating liver partition and portal vein ligation for staged hepatectomy. J Am Coll Surg. 2015;221:717-728.e711.

16. Karoui M, Penna C, Amin-Hashem M, Mitry E, Benoist S, Franc B, Rougier P, Nordlinger B. Influence of preoperative chemotherapy on the risk of major hepatectomy for colorectal liver metastases. Ann Surg. 2006;243:1-7.

17. de Baere T, Teriitehau C, Deschamps F, Catherine L, Rao P, Hakime A, Auperin A, Goere D, Elias D, Hechelhammer L. Predictive factors for hypertrophy of the future remnant liver after selective portal vein embolization. Ann Surg Oncol. 2010;17:2081-9.

18. Sturesson C, Keussen I, Tranberg KG. Prolonged chemotherapy impairs liver regeneration after portal vein occlusion-an audit of 26 patients. Eur J Surg Oncol. 2010;36:358-64.

19. Spelt L, Norman P, Tornqvist L, Tingstedt B, Andersson R. Combined portal vein embolization and preoperative chemotherapy prior to liver resection for colorectal cancer metastases. Scand J Gastroenterol. 2012;47:975-83.

20. Hasselgren K, Malago M, Vyas S, Campos RR, Brusadin R, Linecker M, Petrowsky H, Clavien PA, Machado MA, Hernandez-Alejandro R, et al. Neoadjuvant chemotherapy does not affect future liver remnant growth and outcomes of associating liver partition and portal vein ligation for staged hepatectomy. Surgery. 2017;161:1255-65.

21. Nafidi $O$, Desy D, Letourneau $R$, Cote J, Plasse M, Vandenbroucke F, Roy A, Dagenais M, Lapointe RW. Hypertrophy of the non-embolized liver after chemotherapy. HPB (Oxford). 2009;11:103-7.

22. Covey AM, Brown KT, Jarnagin WR, Brody LA, Schwartz $L$, Tuorto $S$, Sofocleous CT, D'Angelica M, Getrajdman Gl, DeMatteo R, et al. Combined portal vein embolization and neoadjuvant chemotherapy as a treatment strategy for resectable hepatic colorectal metastases. Ann Surg. 2008;247:451-5.

23. Kambakamba P, Linecker M, Alvarez FA, Samaras P, Reiner CS, Raptis DA, Kron P, de Santibanes E, Petrowsky H, Clavien PA, Lesurtel M. Short chemotherapy-free interval improves oncological outcome in patients undergoing two-stage hepatectomy for colorectal liver metastases. Ann Surg Oncol. 2016;23:3915-23.

24. Spelt L, Sparrelid E, Isaksson B, Andersson RG, Sturesson C. Tumour growth after portal vein embolization with pre-procedural chemotherapy for colorectal liver metastases. HPB (Oxford). 2015;17:529-35. 